\title{
REAlidade Virtual e Aumentada (RVA) No ENSINO tÉcnico NAS ÁREAS DE ELÉTRICA, MECÂNICA E CIVIL: UMA REVISÃO DE LITERATURA
}

VIRTUAL AND AUGMENTED REALITY (VAR) IN TECHNICAL TEACHING IN ELECTRICAL, MECHANICAL AND CIVIL AREAS: A LITERATURE REVIEW

REALIDAD VIRTUAL Y AUMENTADA (RVA) EN LA ENSEÑANZA TÉCNICA EN ÁREAS ELÉCTRICAS, MECÁNICAS Y CIVILES: UNA REVISIÓN DE LA LITERATURA

\section{Mariella Maia Quadros \\ (iD) 9}

Mestrado em Engenharia Elétrica

Professora do Ensino Básico,

Técnico e Tecnológico do IFMG

Campus Sabará

mariella.quadros@ifmg.edu.br

\section{Rodrigo Hiroshi Murofushi (iD) 9}

Mestrado em Engenharia

Mecânica

Professor do Ensino Básico,

Técnico e Tecnológico do IFMG

Campus Sabará

rodrigo.hiroshi@ifmg.edu.br

\section{Cláudio Alves Pereira

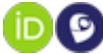

Mestrado em Educação

Professor no Programa de Pós-

Graduação Lato Sensu em

Docência (IFMG Campus

Avançado Arcos)

claudioapessoal@gmail.com

\begin{abstract}
Resumo
Avanços recentes na computação e tecnologias digitais de informação e comunicação (TDIC) têm sido notados nas instituições de ensino e, neste cenário, encontram-se os dispositivos e softwares de Realidade Virtual e Aumentada (RVA). De cunho exploratório, este estudo buscou trabalhos publicados em periódicos e eventos científicos que trataram da utilização de RVA no cotidiano escolar, com foco em cursos das áreas de elétrica, mecânica e civil. $\mathrm{O}$ corpus da pesquisa foi composto por 35 trabalhos que trataram da aplicação de tecnologias de RVA em ambientes escolares, sobressaindo trabalhos desenvolvidos nos cursos superiores e nas disciplinas de matemática e ciências no Ensino Médio. Observou-se a possibilidade de adaptação de trabalhos desenvolvidos em cursos superiores para serem desenvolvidos também em cursos técnicos de nível médio, aproveitando-se as potencialidades destas tecnologias para o exercício da docência e para a formação técnica dos estudantes
\end{abstract}

Palavras-chave: Realidade Virtual. Realidade Aumentada. Ensino Técnico. TDIC.

Recebido em: 12 de julho de 2021.

Aprovado em: 29 de outubro de 2021.

Como citar esse artigo (ABNT):

QUADROS, Mariella Maia; MUROFUSHI, Rodrigo Hiroshi; PEREIRA, Cláudio Alves. Realidade Virtual e Aumentada (RVA) no ensino técnico nas áreas de elétrica, mecânica e civil: uma revisão de literatura. Revista Prática Docente, v. 6, n. 3, e091, 2021.

http://doi.org/10.23926/RPD.2021.v6.n3.e091.id1271 


\begin{abstract}
Recent advances in computing and digital information and communication technologies (DICT) in educational institutions, including Virtual and Augmented Reality (VAR) devices and software stand out. Exploratory in nature, this study searched works published in journals and scientific events that dealt with the use of RVA in everyday school life, focusing on courses in the areas of electrical, mechanics and civil. This research was based on 35 papers that dealt with the application of VAR technologies in school environments, highlighting works developed in higher education courses and in the subjects of mathematics and science in High School. It was observed the possibility of adapting works developed in higher education courses to be developed also in secondary level technical courses, taking advantage of the potential of these technologies for teaching practice and for the technical training of students.
\end{abstract}

Keywords: Virtual Reality. Augmented Reality. Technical Education. DICT.

\title{
Resumen
}

Se destacan los avances recientes en informática y tecnologías digitales de la información y la comunicación (TDIC) en instituciones educativas, incluyendo dispositivos y software de Realidad Virtual y Aumentada (RVA). De naturaleza exploratoria, este estudio buscó trabajos publicados que trataran sobre el uso de RVA en la vida escolar cotidiana, enfocándose en cursos en las áreas de electricidad, mecánica y civil. La investigación se basó en 35 artículos que abordaron la aplicación de tecnologías RVA en entornos escolares, destacando trabajos desarrollados en cursos de educación superior y en las asignaturas de matemáticas y ciencias en Bachillerato. Se observó la posibilidad de adecuar trabajos desarrollados en cursos de educación superior para ser desarrollados también en cursos técnicos de nivel secundario, aprovechando el potencial de estas tecnologías para el ejercicio de la docencia y para la formación técnica de los estudiantes.

Palabras clave: Realidad Virtual. Realidad Aumentada. Educación Técnica. TDIC. 


\section{INTRODUÇÃO}

Com os avanços recentes da computação e das chamadas Tecnologias Digitais de Informação e Comunicação (TDIC), os recursos tecnológicos estão mais presentes e acessíveis ao cotidiano, inclusive no ambiente educacional. A chamada Educação 4.0 pode ser considerada uma nova abordagem que traz à tona a discussão sobre as novas ferramentas do mundo contemporâneo e as habilidades necessárias para que o sujeito se adapte melhor à nova realidade na qual, cotidianamente, estão presentes:

- a conectividade dos sistemas e facilidade de acesso ao conhecimento;

- a inteligência artificial, automação e sistemas robotizados;

- as novas mídias e o Big Data (análise e interpretação de grandes volumes de dados);

- a velocidade da inovação.

Nesse cenário, uma das tecnologias emergentes são os dispositivos e softwares de Realidade Virtual e Aumentada (RVA), amplamente utilizados na indústria do entretenimento e que possuem um grande potencial no uso educacional, em especial nas carreiras técnicas ligadas à tecnologia e engenharia (PAVANI et al., 2017; OLIVEIRA et al., 2019; ROLIM et al., 2019).

Este estudo inspirou-se nas mudanças ocasionadas pela pandemia da Covid-19, precisamente no surgimento de novas vivências e descobertas que o ensino não-presencial trouxe. Dessa forma, professores buscaram alternativas tecnológicas para ministrar aulas teóricas e práticas de forma remota para o ensino técnico e notou-se que a RVA é uma tecnologia promissora para dar mais tangibilidade aos conteúdos ministrados nos componentes curriculares, bem como diminuir o distanciamento transacional ${ }^{1}$ entre alunos e professores.

Sendo assim, este trabalho tem como objetivo fazer uma revisão bibliográfica do uso das ferramentas de Realidade Virtual e Aumentada contextualizadas ao ensino técnico e profissionalizante da área de engenharia, com foco na discussão das potencialidades para a formação técnica do egresso.

O artigo está estruturado da seguinte forma: a Seção 2 apresenta os referenciais teóricos utilizados e definições sobre Realidade Virtual e Realidade Aumentada; na Seção 3 são

\footnotetext{
${ }^{1}$ Segundo Moore (1993), a distância transacional é um conceito que descreve a compreensão e percepção causada pela distância geográfica que tem de ser ultrapassada por professores, alunos e instituições educativas para que ocorra um processo de aprendizagem eficaz, deliberado e planeado. Os procedimentos para ultrapassar esta distância são de natureza interativa e de concepção educativa e, para enfatizar que a distância é pedagógica e não geográfica.
} 
definidos os procedimentos metodológicos; a Seção 4 descreve o mapeamento do campo; e a Seção 5 apresenta as discussões finais.

\section{REFERENCIAIS TEÓRICOS}

Dentre as tecnologias já criadas e aperfeiçoadas pelo homem, a informática e a digitalização do mundo evoluem em uma velocidade antes nunca vista (BARRILLI; CUNHA, 2009). Potencializada pelos avanços da microeletrônica e recursos de softwares, as Tecnologias Digitais de Informação e Comunicação (TDIC) estão cada vez mais presentes no cotidiano do ser humano; impulsionadas pela internet, elas têm transformado as formas de pensar e de expressar, de aprender e de comunicar em todas as áreas e atividades (LIMA, 2017).

No campo da educação, as TDICs podem contribuir na integração de práticas pedagógicas inovadoras, que favorecem a autonomia dos alunos, além de permitir acesso, construção, intercâmbio de ideias e conteúdos disciplinares em diversos formatos como textos, imagens, sons, simulações, dentre outros (STERN, 2008 apud LIMA, 2017). De acordo com Barrilli e Cunha (2009), boas práticas pedagógicas inovadoras buscam integrar teoria e prática, abordando soluções a problemas reais do cotidiano e privilegia uma didática humanista, integradora, baseada na pesquisa, reflexão e tomada de decisão.

Dentre as metodologias de ensino amplamente estudadas na atualidade, está a utilização de metodologias ativas que buscam inserir a experimentação no processo de ensinoaprendizagem dos alunos. Esta tem se mostrado uma das estratégias mais eficazes, pois os conteúdos vistos e discutidos em sala de aula são assimilados com mais facilidade pelos estudantes quando a experiência educativa é desafiadora e significativa (ALMEIDA et al., 2018). Uma experiência educativa mais rica e envolvente possui o potencial de produzir maior significado para o aprendiz, pois é por meio da experimentação que o aluno desenvolve sua curiosidade, explora suas afinidades, entra em contato com dificuldades, problemas, desafios, instrumentos, entre outros. Essa dinâmica é o que possibilita ao educando desenvolver habilidades e competências necessárias que farão parte do seu cotidiano ao ingressar no mercado de trabalho e constituir-se como cidadão.

Nessa linha de pensamento, segundo Ausburn e Ausburn (2018), muitas tecnologias visuais influenciaram e aprimoraram as instruções em educação e melhoraram a capacitação de especialistas e técnicos. Algumas dessas ferramentas são as tecnologias visuais, as quais trouxeram para as salas de aula e laboratórios um realismo que melhorou a compreensão, 
aumentou o desempenho do aprendizado e reduziu o tempo de treinamento. Essas tecnologias compõem uma família de TDIC.

Dentre as novas TDIC, a Realidade Aumentada (RA) desponta como uma das mais promissoras em publicações de periódicos e eventos científicos, e com potencial de utilização no âmbito educacional (HAMILTON, 2011). Segundo Tori, Kirner e Siscoutto (2006), a RA pode ser conceituada como "[...] o enriquecimento do ambiente real com objetos virtuais, usando algum dispositivo tecnológico, funcionando em tempo real" (p. 25).

Outra TDIC que pode contribuir para aumentar o interesse e a motivação dos alunos com alto potencial para aprimorar a experiência de aprendizagem é a Realidade Virtual (RV). O termo RV já passou por contínuas mudanças desde que foi apresentada na década de 1960 e, atualmente, a RV se refere a uma variedade de experiências desenvolvidas em computador, desde ambientes totalmente imersivos com equipamentos HMD (capacetes de realidade virtual, do inglês Head Mounted Displays), entrada auditiva, ativação por voz, luvas de dados (e até vestimentas com biossensores para entrada sensorial avançada e biofeedback), a imagens realistas baseadas em simulações computadorizadas. No entanto, em todas as suas formas, a RV é basicamente uma maneira de simular ou replicar um ambiente tridimensional e dar aos usuários a sensação de "estar lá", assumir o controle e interagir pessoalmente com o ambiente por meio de seus próprios corpos e sentidos (AUSBURN; AUSBURN, 2004).

Dessa forma, de acordo com Tori e Kirner (2006, apud TORI; HOUNSELL, 2018) a realidade virtual pode ser definida como uma interface avançada do usuário utilizada para o acesso a aplicações executadas no computador. Além disso, possui como características principais a visualização de ambientes tridimensionais em tempo real, a movimentação nesses ambientes e a interação com elementos pertencentes a eles. A realidade aumentada também inclui no ambiente físico objetos criados computacionalmente, possibilitando a coexistência de objetos reais e virtuais - considerada uma vertente da realidade virtual. Ou seja, na RV o usuário é transportado para um ambiente virtual, tendo como consequência a abstração completa do ambiente físico e local, enquanto na RA são mantidas as referências para o ambiente real e elementos virtuais são transportados para o espaço local do usuário (TORI; HOUNSELL, 2018).

Sendo assim, atualmente, o uso de Realidade Virtual e Aumentada (RVA) vem sendo amplamente estudado no aprimoramento dos processos de ensino e aprendizagem, bem como no engajamento dos alunos às novas práticas pedagógicas (FRANÇA; SILVA, 2019; SOUSA 
et al., 2019; FRANÇA; SILVA, 2020). Porém, nota-se uma grande divergência de práticas e trabalhos desenvolvidos com foco em cursos superiores e naqueles que têm foco nos cursos técnicos e nas disciplinas da formação geral de matemática e ciências para o ensino médio, principalmente as ligadas à formação de profissionais inseridos no contexto da chamada Indústria 4.0 ou $4^{\mathrm{a}}$ revolução industrial.

De acordo com Lorenz et al. (2015) do BCG (The Boston Consulting Group), nove tecnologias avançadas são bases para a Indústria 4.0: robôs autônomos, simulação, integração horizontal e vertical de sistemas, a internet das coisas, segurança da informação, nuvem de dados, manufatura aditiva, realidade aumentada, big data e analytics.

O estudo realizado por Silva et al. (2019) buscou entender como as instituições de educação técnica profissionalizante estão se preparando para formar profissionais capazes de atuar com as principais tecnologias industriais. Registra-se que a RVA não foi citada pelos professores das unidades das instituições de ensino entrevistados como um recurso didático presente no cotidiano de estudo das turmas de ensino técnico.

Outro trabalho, dessa vez apresentado em Exame e Estúdio ABC (2016) com o auxílio da SIEMENS (empresa alemã dentre as maiores no ramo de tecnologia industrial do mundo), ao abordar o perfil do profissional para a indústria 4.0, expôs que técnicos deixarão de exercer funções repetitivas, como o encaixe de uma peça em um smartphone, por exemplo, e isso não significa que os funcionários serão eliminados das linhas de produção. Segundo a publicação, esses profissionais ficarão concentrados em tarefas estratégicas e no controle de projetos; para que as indústrias possam contar com profissionais com essa qualificação, a capacitação técnica deve ser aprimorada em seus setores. Assim, formas de ensino e treinamento que fazem uso de simulações por meio da RVA tornaram-se especialmente importantes.

A próxima seção apresentará as escolhas metodológicas dos pesquisadores para este estudo exploratório.

\section{Procedimentos Metodológicos}

Esta pesquisa possui caráter bibliográfico exploratório baseado na revisão sobre realidade virtual e aumentada aplicadas no ensino dos cursos técnicos da área de engenharia, especificamente das engenharias mecânica, elétrica e civil.

Foram realizadas buscas na base de periódicos Google Scholar durante o mês de outubro de 2020, com termos descritores referentes à Realidade Virtual e à Realidade Aumentada (ver Tabela 1) na área de engenharia ou de cursos técnicos e profissionalizantes nesta mesma área. 
Como recorte temporal, foram consideradas publicações dos últimos 10 anos (2010-2020) e artigos publicados na Língua Portuguesa, já que o interesse dos pesquisadores era identificar a utilização de sistemas de RV e RA na educação brasileira. Além disso, foram realizadas buscas nos anais do Congresso Brasileiro de Educação em Engenharia (COBENGE) com os descritores "Realidade Aumentada" e "Realidade Virtual" considerando publicações dos anos de 2017, 2018 e 2019 e publicações da Revista Brasileira de Ensino de Ciência e Tecnologia (RBECT), com os descritores "Realidade Virtual" e "Realidade Aumentada" dos últimos 10 anos (20102020). A base das publicações, o período de busca das publicações, os descritores utilizados e a quantidade de trabalhos encontrados podem ser visualizados no Quadro $1^{2}$.

Quadro 1 - nformações sobre as buscas realizadas

\begin{tabular}{|c|c|c|c|}
\hline $\begin{array}{l}\text { Base de periódicos } \\
\text { (período de } \\
\text { publicação) }\end{array}$ & Descritores & $\begin{array}{c}\text { Quantidade de } \\
\text { Trabalhos } \\
\text { Encontrados }\end{array}$ & $\begin{array}{c}\text { Quantidade de } \\
\text { trabalhos pré- } \\
\text { selecionados }\end{array}$ \\
\hline $\begin{array}{l}\text { Google Scholar } \\
\text { (2010-2020) }\end{array}$ & "Realidade Virtual”, "Educação”, "Técnica” & 12.300 & 0 \\
\hline $\begin{array}{l}\text { Google Scholar } \\
(2010-2020)\end{array}$ & "Realidade Virtual", "Educação", "Profissional" & 13.600 & 0 \\
\hline $\begin{array}{l}\text { Google Scholar } \\
\quad(2010-2020)\end{array}$ & "Realidade Aumentada", "Educação", "Técnica" & 4.120 & 0 \\
\hline $\begin{array}{c}\text { Google Scholar } \\
\text { (2010-2020) }\end{array}$ & $\begin{array}{l}\text { "Realidade Aumentada", "Educação", } \\
\text { "Profissional" }\end{array}$ & 4.620 & 0 \\
\hline $\begin{array}{c}\text { Google Scholar } \\
(2010-2020)\end{array}$ & "Realidade Virtual”, "Educação", "Engenharia" & 7.330 & 4 \\
\hline $\begin{array}{l}\text { Google Scholar } \\
\text { (2010-2020) }\end{array}$ & $\begin{array}{l}\text { "Realidade Aumentada", "Educação", } \\
\text { "Engenharia" }\end{array}$ & 3.130 & 2 \\
\hline $\begin{array}{l}\text { Google Scholar } \\
\text { (2010-2020) }\end{array}$ & $\begin{array}{l}\text { "Realidade Aumentada", "Educação", } \\
\text { "Ciências" }\end{array}$ & 5.170 & 6 \\
\hline $\begin{array}{c}\text { Google Scholar } \\
(2010-2020) \\
\end{array}$ & "Realidade Virtual”, "Educação”, “Ciências” & 14.700 & 1 \\
\hline $\begin{array}{l}\text { COBENGE (2017- } \\
\text { 2019) }\end{array}$ & "Virtual" & 18 & 12 \\
\hline $\begin{array}{l}\text { COBENGE (2017- } \\
\text { 2019) }\end{array}$ & "Realidade Aumentada" & 8 & 8 \\
\hline $\begin{array}{c}\text { RBECT (2010- } \\
2020) \\
\end{array}$ & "Realidade Aumentada" & 3 & 2 \\
\hline $\begin{array}{c}\text { RBECT } \\
(2010-2020)\end{array}$ & "Realidade Virtual" & 3 & 0 \\
\hline
\end{tabular}

Fonte: Elaborado pelos autores (2021).

A partir dos artigos encontrados, foram realizadas outras filtragens com o objetivo de selecionar apenas os mais relevantes e relacionados ao tema de interesse, por meio da leitura

\footnotetext{
2 É importante ressaltar que também foram realizadas pesquisas no Portal de Periódicos da Coordenação de Aperfeiçoamento de Pessoal de Nível Superior (Capes), tendo sido obtidos apenas 9 artigos relacionados à educação no ensino técnico ou superior nas áreas de civil, elétrica e mecânica. Entretanto, todos os trabalhos retornados por este portal já tinham sido encontrados em outras bases e, portanto, essa busca não foi incluída no Quadro 1.
} 
dos títulos, resumos (abstract) e palavras-chave. Para isso, foram aplicados filtros de exclusão, e os artigos fora da área técnica de engenharia (como publicações na área de saúde, de música, de artes, de psicologia, entre outros) e aqueles não configuravam trabalhos direcionados ao ensino médio, técnico ou superior, foram eliminados da seleção.

Em relação aos trabalhos direcionados ao ensino médio, apenas aqueles com aplicação em matemática e ciências foram selecionados, por estarem diretamente relacionados com as disciplinas dos cursos técnicos. É importante ressaltar que mesmo as buscas realizadas com os descritores específicos do Quadro 1, muitos artigos com abordagens distantes da investigada foram retornados pelas bases, principalmente na base do Google Scholar. Isso se deve ao fato de as palavras "Engenharia", "Técnica", "Profissional" e "Ciências" apareceram nos trabalhos em outros contextos, não diretamente relacionados com a educação em engenharia, técnica e profissional, e em ciências. Por exemplo, a referência à utilização de Engenharia de Software para desenvolvimento dos recursos de RVA e às técnicas de procedimentos realizados e descritos nos trabalhos. Ou seja, apesar de os descritores estarem presentes no texto, não representam o tema principal do trabalho.

Um outro filtro de exclusão utilizado foi a eliminação de artigos que não possuíam identificação do periódico ou evento científico em que foram publicados, isto é, que podem ser artigos que não foram avaliados antes de sua publicação e que podem ter sido disponibilizados pelos próprios autores. Além disso, foram eliminados os artigos de periódicos com classificação inferior a Qualis C e artigos de eventos científicos locais (apenas artigos de eventos de abrangência nacional e internacional foram selecionados).

Um último filtro utilizado pelos pesquisadores foi a eliminação de trabalhos repetidos, foram retirados os trabalhos que apareceram em mais de uma busca do Quadro 1, sendo escolhidos apenas os da primeira ocorrência. Dessa forma, embora o número de documentos encontrados seja muito alto, a maioria foi descartada pelos filtros de exclusão. Em relação ao Google Scholar, para todos os descritores buscados, é possível notar que a partir da quinta página de busca (dez resultados por página), os resultados encontrados já não possuíam nenhuma relação com o tema investigado.

A partir da aplicação dos filtros de exclusão descritos anteriormente, restaram apenas os trabalhos diretamente relacionados à área de interesse, obtendo-se os números apresentados na última coluna do Quadro 1 para cada busca realizada. Assim, restaram 35 artigos para compor o portfólio final, que foram lidos na íntegra e que serão investigados e analisados com mais 
detalhes na próxima seção deste trabalho. É possível notar que, apesar de as buscas retornarem um número elevado de trabalhos, apenas uma pequena parte deles possuem contribuição significativa sobre RVA na educação técnica e nas engenharias mecânica, elétrica e civil.

\section{MAPEAMENTO DO CAMPO E DiscuSSõeS}

Após aplicados os filtros de busca e de exclusão, foram selecionados 35 artigos; todos eles possuem aplicação de RV ou RA ou ambas as tecnologias, RVA, voltadas para a educação da área de engenharia, em cursos de nível técnico integrado ou cursos superiores.

\subsection{MAPEAMENTO 1: ÁREAS DE APLICAÇÃo DA ENGENHARIA (TÉCNICA):}

A partir da análise dos artigos do portfólio final, foi possível perceber algumas tendências sobre a utilização da RVA em contexto educacional, tendo se destacado o uso da realidade aumentada por diferentes áreas de aplicação. Dessa forma, o primeiro mapeamento realizado foi baseado na área de aplicação da tecnologia RVA e no nível de ensino empregado, baseado nestes critérios:

- Matemática e Ciências: apresenta ferramentas de RVA e estudos de casos aplicados a disciplinas de matemática e ciências (química, física, biologia, geografia, entre outras) voltadas para o ensino médio.

- Básica: apresenta ferramentas de RVA e estudos de casos aplicados a componentes curriculares do ciclo básico de cursos profissionalizantes da área de engenharia como, por exemplo, desenho técnico, mecânica, resistência de materiais, meio ambiente, topografia, entre outras. Esta classificação foi subdivida em duas subcategorias, uma voltada para componentes curriculares básicos de cursos técnicos e outra voltada para o ciclo básico de cursos superiores que podem pertencer à matriz curricular de qualquer área da mecânica, elétrica ou civil. Vale ressaltar que trabalhos de RVA relacionados ao ensino de física no ensino superior também foram incluídos na classificação.

- Mecânica: apresenta ferramentas de RVA e estudos de casos aplicados na área de mecânica. Esta classe foi subdividida em duas subcategorias, uma voltada para o ensino técnico e outra dirigida para componentes curriculares de cursos superiores.

- Elétrica: apresenta ferramentas de RVA e estudos de casos aplicados na área de elétrica, eletrônica ou automação. Esta classe foi subdividida em duas subcategorias, uma voltada para o ensino técnico e outra dirigida para componentes curriculares de cursos superiores.

- Civil: apresenta ferramentas de RVA e estudos de casos aplicados na área de construção civil. Esta classe foi subdividida em duas subcategorias, uma voltada para o ensino técnico e outra dirigida para componentes curriculares de cursos superiores. 
- RVA "puro": discute sobre tipos de tecnologias RVA empregadas, softwares e características de desenvolvimento de dispositivos de RVA aplicados em educação, não possuindo aplicação em uma determinada área específica.

Estabelecidos esses critérios, realizou-se, posteriormente, a classificação dos trabalhos selecionados. O portfólio obtido está apresentado no Quadro 2 e nos gráficos da Figura 1. Ressalta-se que, na organização do Quadro 2, um mesmo trabalho pode estar vinculado a mais de uma área de aplicação e ser contabilizado mais de uma vez devido à sua aplicabilidade em mais de uma área.

Quadro 2 - Classificação dos trabalhos do portfólio em áreas, nível educacional e respectivas quantidades

\begin{tabular}{|c|c|c|c|}
\hline Área & $\begin{array}{ll}\text { Autores } \\
\end{array}$ & Quantidade & Total \\
\hline $\begin{array}{l}\text { Matemática e } \\
\text { Ciências } \\
\text { (Ensino Médio) }\end{array}$ & $\begin{array}{l}\text { Barbosa e Carvalho (2017); } \\
\text { Dernadin e Manzano (2017); } \\
\text { França e Silva (2019); } \\
\text { França e Silva (2020); } \\
\text { Leite (2019); } \\
\text { Martins, Oliveira e Guimarães (2013); } \\
\text { Merino et al. (2018); } \\
\text { Oliveira e Manzano (2016); } \\
\text { Queiroz, Moura e Souza (2019); } \\
\text { Viegas, Vieira e Silva (2012)*. }\end{array}$ & 10 & 10 \\
\hline $\begin{array}{l}\text { Básica } \\
\text { (Técnico) }\end{array}$ & - & 0 & \\
\hline $\begin{array}{c}\text { Básica } \\
\text { (Superior) }\end{array}$ & $\begin{array}{l}\text { Herpich } \text { et al. (2020); } \\
\text { Parcianello e Lemos (2018); } \\
\text { Scortegagna e Gonçalves (2018); } \\
\text { Silva (2019); } \\
\text { Silva et al. (2015); } \\
\text { Souza, Morais e Carvalho (2018); } \\
\text { Viegas, Vieira e Silva (2012)*. }\end{array}$ & 7 & 7 \\
\hline $\begin{array}{l}\text { Elétrica } \\
\text { (Técnico) }\end{array}$ & $\begin{array}{l}\text { Oliveira et al. }(2019)^{*} ; \\
\text { Pavani et al. }(2017)^{*} \\
\text { Rolim } \text { et al. }(2019)^{*} \text {. }\end{array}$ & 3 & \\
\hline $\begin{array}{c}\text { Elétrica } \\
\text { (Superior) }\end{array}$ & $\begin{array}{l}\text { Araújo e Carreiro (2017); } \\
\text { Almeida et al. (2018); } \\
\text { Correa et al. (2019); } \\
\text { Ferreira, Conceição e Fonseca (2019); } \\
\text { Gomes Junior, Cardoso, Lamounier Junior (2010); } \\
\text { Pavani, Pozzer e Colusso (2019)*; } \\
\text { Silva, Sá Júnior e Azevêdo (2018); } \\
\text { Vilar, Araújo e Costa Junior (2017)*. }\end{array}$ & 8 & 11 \\
\hline $\begin{array}{l}\text { Mecânica } \\
\text { (Técnico) }\end{array}$ & $\begin{array}{l}\text { Oliveira et al. (2019)*; } \\
\text { Pavani et al. }(2017)^{*} ; \\
\text { Rolim } \text { et al. }(2019)^{*} .\end{array}$ & 3 & 6 \\
\hline $\begin{array}{l}\text { Mecânica } \\
\text { (Superior) }\end{array}$ & $\begin{array}{l}\text { Pavani, Pozzer e Colusso (2019)*; } \\
\text { Sena, Rocha e Nascimento (2018); } \\
\text { Vilar, Araújo e Costa Junior (2017)*. }\end{array}$ & 3 & 0 \\
\hline $\begin{array}{c}\text { Civil } \\
\text { (Técnico) }\end{array}$ & - & 0 & 5 \\
\hline
\end{tabular}




\begin{tabular}{|c|l|c|c|}
\hline $\begin{array}{c}\text { Civil } \\
\text { (Superior) }\end{array}$ & $\begin{array}{l}\text { Alberte e Pereira (2019); } \\
\text { Fortuna } \text { et al. (2019); } \\
\text { Lima } \text { et al. (2019); } \\
\text { Santos } \text { et al. (2013). } \\
\text { Stange e Scheer (2012); }\end{array}$ & 5 & \\
\hline RVA “puro" & $\begin{array}{l}\text { Lopes } \text { et al. (2019); } \\
\text { Queiroz, Tori e Nascimento (2017). }\end{array}$ & 2 & 2 \\
\hline
\end{tabular}

Fonte: Elaborado pelos autores (2021).

* Artigos com mais de uma área de atuação e que não estão incluídos na contagem total.

Já a Figura 1 apresenta índices percentuais dos trabalhos de acordo com a área de atuação e com a separação da amostra em nível superior e ensino técnico de nível médio.

Figura 1 - Gráficos dos percentuais dos trabalhos em relação à área de atuação: (a) sem divisão entre aplicação no nível técnico e superior; (b) com a classificação entre aplicação no nível técnico e superior

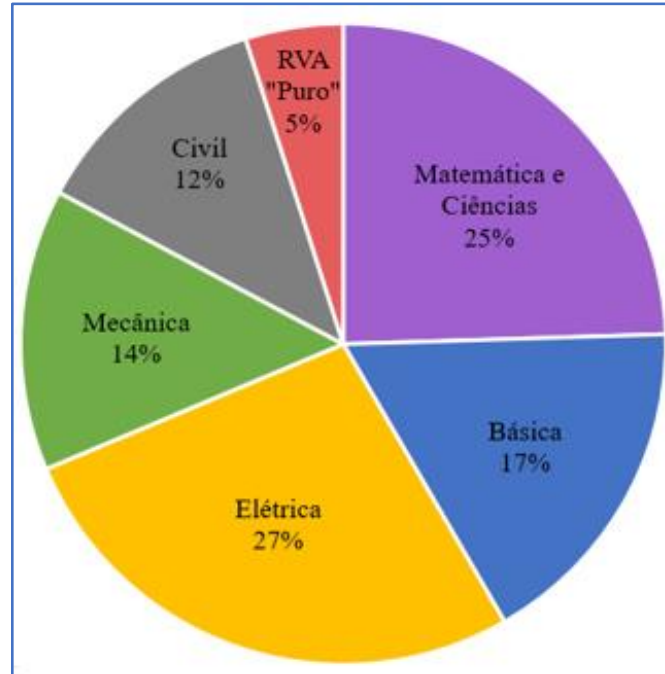

(a)

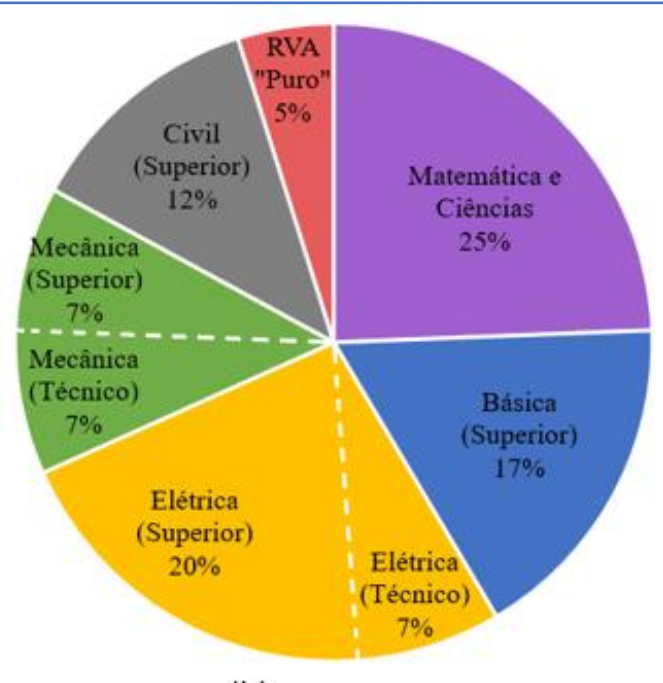

(b)

Fonte: Elaborada pelos autores (2021).

Pela análise do gráfico apresentado pela Figura 1(a), nota-se que as maiores aplicações de RVA ocorrem na área de elétrica (27\%) e no ensino de matemática e ciências (25\%), seguido pelas aplicações em disciplinas básicas (17\%) e mecânica (14\%). Finalizando a sequência, temse a área de civil (12\%) e estudos sobre RVA aplicados à educação de maneira geral (5\%).

Observando o gráfico da Figura 1(b), nota-se que a RVA é aplicada majoritariamente em cursos superiores perante os cursos técnicos nas áreas de aplicação determinadas neste trabalho, exceto para matemática e ciências e RVA "puro". Assim, é possível fazer outro mapeamento a respeito do nível de ensino em que essa tecnologia é comumente aplicada. 


\subsection{MAPEAMENTO 2: NÍVEIS DE ENSINO}

Outra análise realizada teve foco no nível de ensino em que a RVA é aplicada, conforme descrito nos trabalhos presentes no portfólio. Dessa forma, foi proposta a seguinte classificação dos trabalhos:

- Superior: uso de RVA em cursos de nível superior das áreas das engenharias mecânica, elétrica e civil;

- Técnico: uso de RVA para cursos de nível técnico das áreas das engenharias mecânica, elétrica e civil;

- Médio (Matemática e Ciências): uso de RVA para o ensino de matemática e ciências, para o ensino de nível médio. Observa-se que este nível pode ser aplicado para cursos técnicos cujo ensino médio é integrado ao técnico.

- RVA "puro": trabalho cuja aplicação está relacionada ao ensino, porém não possui aplicação em um nicho específico, por exemplo, trabalhos sobre revisão bibliográfica de RVA ou comparação de softwares ou ferramentas de RVA voltadas para uso no ensino.

O Quadro 3 e o gráfico da Figura 2 apresentam a classificação realizada pelos pesquisadores em relação à divisão dos trabalhos por níveis de ensino.

Quadro 3 - ivisão dos trabalhos do portfólio em níveis de ensino

\begin{tabular}{|l|c|c|}
\hline \multicolumn{1}{|c|}{ Nível de aplicação } & Quantidade de artigos & Percentual \\
\hline Superior & 21 & $58 \%$ \\
\hline Técnico & 3 & $8 \%$ \\
\hline Médio (Matemática e Ciências) & 10 & $28 \%$ \\
\hline RVA "puro" & 2 & $6 \%$ \\
\hline TOTAL & $36^{*}$ & $100 \%$ \\
\hline
\end{tabular}

Fonte: Dados da pesquisa (2021).

* Ressalta-se que o trabalho de Viegas, Vieira e Silva (2012), foi contabilizado duplamente devido à sua aplicação em dois níveis de ensino diferentes (Ensino Médio e Superior). 
Figura 2 - Gráfico da classificação percentual dos trabalhos do portfólio em relação ao nível de ensino aplicado (Médio, Técnico e Superior)

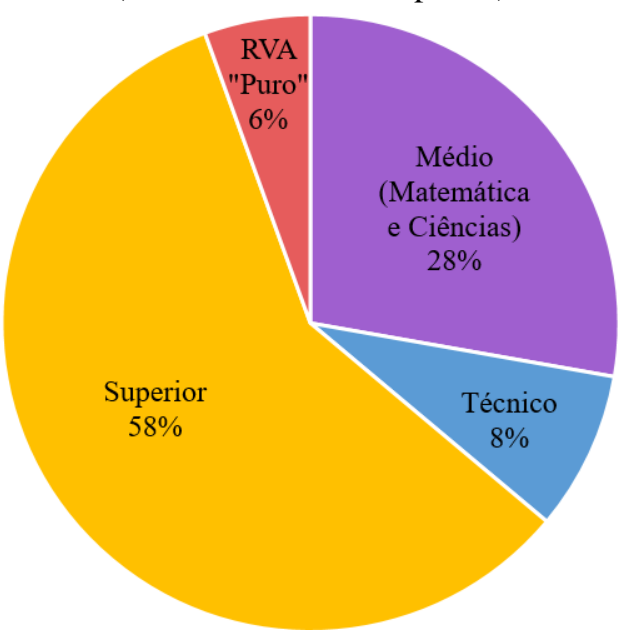

Fonte: Elaborada pelos autores (2021).

Por meio da análise do conjunto dos trabalhos selecionados, notou-se que a maior parcela do portfólio aplica RVA no nível superior de ensino (58\%), seguido pelo nível médio (28\%) e, posteriormente, o nível técnico (8\%). Entretanto, é importante ressaltar que, pela leitura dos trabalhos, as práticas desenvolvidas e assuntos estudados estão diretamente relacionados e possuem aplicabilidade em cursos técnicos, apresentando, portanto, potencial de aplicação também neste nível de ensino. Dessa forma, fica evidente a subutilização da RVA nas áreas técnicas das áreas de civil, elétrica e mecânica.

\subsection{REVISÃO E TRABALHOS RELEVANTES SOBRE O USO DE RVA NO ENSINO E APLICABILIDADE NO ENSINO TÉCNICO}

De acordo com o Quadro 1, em relação a aplicações em cursos técnicos da área de elétrica, destacam-se os trabalhos de Pavani et al. (2017), Oliveira et al. (2019) e Rolim et al. (2019). O primeiro trabalho apresenta uma bancada didática virtual de pneumática, que é muito comum em disciplinas de automação, presentes em diversos cursos técnicos da área da elétrica e da mecânica. Por sua vez, os dois últimos artigos apresentam o desenvolvimento e a utilização de um ambiente para simulação imersiva para capacitação técnica na área de manutenção eletromecânica ferroviária e que, devido à sua natureza, também podem ser classificados como da área da mecânica.

Os trabalhos relacionados ao ensino técnico da área de mecânica são os de Oliveira $e t$ al. (2019), Pavani et al. (2017) e Rolim et al. (2019) que também abordam o ensino técnico na área de elétrica e descritos anteriormente. 
Apesar da RA ser utilizada como ferramenta de visualização para a metodologia Building Information Modeling (BIM) na arquitetura e engenharia, nenhum artigo foi encontrado com aplicações educacionais na área técnica de civil, o que indica a RA ainda é pouco explorada em seus cursos.

A partir das análises aqui apresentadas, percebe-se uma grande lacuna na literatura científica sobre o desenvolvimento ou estudo de RVA na educação técnica, tecnológica e profissionalizante nas áreas de elétrica, mecânica e civil, já que apenas uma parte dos trabalhos selecionados $(8 \%)$ apresenta uma abordagem direcionada à formação do estudante de nível técnico. A quantidade de trabalhos ainda leva em consideração aqueles que se encaixam em mais de uma categoria, o que mostra que o investimento financeiro e até mesmo de tempo empregado no desenvolvimento e aplicação dessa tecnologia devem ser aumentados e incentivados de maneira a proporcionar uma capacitação compatível com as exigências da Indústria 4.0.

Por outro lado, percebe-se um número relativamente maior de trabalhos voltados para disciplinas de matemática e ciências do ensino médio (28\%), como mostra o Quadro 2. Dentre eles, podem ser destacados os artigos de Dernadin e Manzano (2017), França e Silva (2020), Oliveira e Manzano (2016) e Viegas, Vieira e Silva (2012), que apresentam aplicações de RA ou RV no ensino de física. Ainda, Barbosa e Carvalho (2017) e Martins, Oliveira e Guimarães (2013) demonstram o uso de RA no ensino de geometria.

Apesar de os artigos abordarem o uso de RVA em disciplinas do ensino médio regular, essa tecnologia pode ser utilizada nas disciplinas da formação geral dos cursos técnicos integrados, inclusive possibilitando a interdisciplinaridade com algumas áreas técnicas. Vários conceitos aprendidos em matemática e ciências estão diretamente relacionados com os conteúdos técnicos e uma contextualização entre eles pode enriquecer a aprendizagem do aluno, principalmente com a utilização de recursos como a RVA. Alguns exemplos são a utilização de conceitos de geometria em disciplinas de desenho técnico, de eletromagnetismo em disciplinas de eletrônica analógica e de potência, de química em disciplinas de ciência dos materiais, entre outros.

Com uma diferença ainda maior, o número de trabalhos selecionados referentes a conteúdos e disciplinas dos cursos superiores de engenharia equivalem à maioria dos trabalhos levantados (58\%), o que evidencia um maior interesse e investimento nesse nível de educação. Contudo, nota-se por meio da análise dos artigos que, apesar de suas aplicações de RVA terem 
sido direcionadas para os cursos de engenharia naqueles estudos, várias delas podem ser implementadas com nenhuma ou poucas modificações em turmas do ensino técnico das áreas de elétrica, mecânica ou civil. Alguns exemplos são os trabalhos de Araújo e Carreiro (2017), Correa et al. (2019), Pavani, Pozzer e Colusso (2019), Almeida et al. (2018) e Ferreira, Conceição e Fonseca (2019); nesses, apresentam-se aplicações na área de elétrica direcionadas para automação industrial, eletrotécnica e eletrônica analógica, que são conteúdos encontrados em disciplinas de diversos cursos técnicos da área. Outros exemplos, da área de mecânica, são os trabalhos de Pavani, Pozzer e Colusso (2019) e Vilar, Araújo e Costa Junior (2017) aplicados no ensino de pneumática e robótica (também classificados em elétrica), adaptáveis para o ensino técnico. Por último, podem ser citados os trabalhos de Alberte e Pereira (2019) e Fortuna et al. (2019), que podem ser estendidos para cursos da formação técnica na área de construção civil.

\section{CONSIDERAÇÕES FINAIS}

Com os avanços tecnológicos dos últimos anos e principalmente com o surgimento da Indústria 4.0, as exigências de formação e capacitação dos profissionais em relação às novas tecnologias têm se tornado cada vez maiores. Nesse contexto, este trabalho buscou investigar e analisar a literatura acadêmica que trata sobre a utilização e implementação de Realidade Virtual e Realidade Aumentada no ensino técnico na área de engenharias, especificamente de elétrica, mecânica e civil. Para isso, foram realizadas buscas com os principais descritores relacionados ao tema e aplicados filtros de exclusão, com o objetivo de selecionar apenas os artigos mais relevantes e que realmente poderiam contribuir para uma análise significativa.

Após a aplicação dos filtros, foram selecionados 35 trabalhos e realizados dois mapeamentos: (i) das áreas de aplicação da engenharia (técnica); e (ii) dos níveis de ensino aplicado. No primeiro deles, os artigos foram mapeados de acordo com a área de aplicação da engenharia, pelos critérios de Matemática e Ciências, Área Básica, Mecânica, Elétrica, Civil e RVA “puro". De acordo com o mapeamento, constatou-se que $27 \%$ dos trabalhos são da área de elétrica, $14 \%$ da mecânica e $12 \%$ de civil. Ou seja, pouco mais da metade dos artigos apresenta estudos e aplicações de RVA em conteúdos e disciplinas técnicas da área de engenharia. Os demais trabalhos são dedicados à utilização dessa tecnologia em matemática e ciências (25\%), aos conteúdos básicos (17\%) e RVA “puro” (5\%). Já o segundo mapeamento classificou os trabalhos em Superior, Técnico, Médio e RVA “puro", verificando-se que apenas três artigos $(8 \%)$ se referem à utilização e estudo de RVA na formação técnica de nível médio nas áreas de elétrica, mecânica e civil. Os trabalhos restantes são majoritariamente direcionados 
a estudos desenvolvidos em cursos superiores de engenharia (58\%), uma quantia significativa aos conteúdos e disciplinas do ensino médio (28\%) e uma pequena quantidade sobre RVA "puro" (6\%).

Vale mencionar que a tecnologia RVA pode ser utilizada de forma interdisciplinar tanto em disciplinas técnicas quanto na formação geral, em especial em matemática e ciências. Isto porque o ambiente virtual construído pode ser aproveitado sob diferentes perspectivas, por exemplo, a matéria de geometria plana e espacial, ministrada em matemática, pode também ser aplicada em desenho técnico para cursos profissionalizantes nas áreas de mecânica, elétrica e civil. Outro exemplo é a eletrodinâmica estudada em física, que pode ser contextualizada na área de instalações elétricas. Outro caso é a hidrostática estudada na física, que pode ser aplicada ao estudo de sistemas hidráulicos e pneumáticos. Finalizando os exemplos, mas não as possibilidades de trabalhos interdisciplinares, a estática e a dinâmica, também objetos de estudo em física, formam a base para as disciplinas de resistência, análise estrutural e teoria das estruturas.

Portanto, é possível concluir que a RVA é pouco explorada como recurso a ser utilizado nos processos de ensino e aprendizagem de cursos técnicos da área de engenharia, principalmente quando os resultados são comparados com estudos no ensino superior de engenharia e com disciplinas da formação geral de nível médio. Também é importante ressaltar que vários dos artigos direcionados para o ensino superior analisados neste trabalho podem ser aplicados com nenhuma ou poucas modificações para o ensino técnico de nível médio. Assim, é importante que as instituições de ensino técnico e profissionalizante realizem investimentos em novas tecnologias como a RVA, que tem se mostrado um recurso muito eficiente no ensino e treinamento técnico em diferentes áreas do conhecimento, inclusive nas engenharias elétrica, mecânica e civil.

\section{REFERÊNCIAS}

ALBERTE, Elaine Pinto Varela; PEREIRA, Mariana Henry. Uma Experiência de Aprendizagem das Etapas Executivas de uma Construção Através de Maquete Virtual. In: COBENGE, XLVII, 2019, Fortaleza-CE. Anais [...]. Brasília: ABENGE, 2019, v. 1, s.p.

ALMEIDA, Bruno Ricardo et al. Realidade Aumentada Aplicada às Práticas Laboratoriais nos Cursos de Engenharia. In: XLVI Congresso Brasileiro de Educação em Engenharia e $1^{\circ}$ Simpósio Internacional de Educação em Engenharia (COBENGE), 2018, Salvador-BA. Anais [...]. Brasília: ABENGE, 2018, v. 1, s.p. 
ARAUJO, Felipe A. Torres; CARREIRO, Francisco Borges. Laboratório Semi-Virtual para Aprendizagem Interativa de Automação e Controle de Processos Industriais. In: COBENGE, XLV, 2017, Joinville-SC. Anais [...]. Brasília: ABENGE, 2017, v. 1, s.p.

AUSBURN, Lynna J.; AUSBURN, Floyd B. Desktop virtual reality: A powerful new technology for teaching and research in industrial teacher education. Journal of Industrial Teacher Education, v. 41, n. 4, p. 1-16, 2004.

BARBOSA, Jorge William Sandora; CARVALHO, Carlos Vitor de Alencar. Geotransform3D: Objeto Computacional em Realidade Aumentada para apoio ao Ensino da Matemática. Revista de Educação, Ciências e Matemática (RECM), v. 1, p. 226-239, 2017.

BARRILLI, Elomar Castilho; CUNHA, Gerson Gomes. A tecnologia de realidade virtual: recurso real para potencializar a educação. Virtual Reality and Scientific Visualization Journal, v. 2, n. 2, p. 1-16, 2009.

CORREA, Luiz Fernando de Oliveira et al. Mundo Virtual 3D Estreitando Relações TeóricoPráticas nas Instituições de Ensino. In: COBENGE, XLVII, 2019, Fortaleza-CE. Anais [...]. Brasília: ABENGE, 2019, v. 1, s.p.

DENARDIN, Luciano; MANZANO, Ramón Cid. Desenvolvimento, utilização e avaliação da realidade aumentada em aulas de física. Revista Novas Tecnologias na Educação, [S.L.], v. 15, n. 2, 10 jan. 2018. Universidade Federal do Rio Grande do Sul.

http://dx.doi.org/10.22456/1679-1916.79258

EXAME; ESTÚDIO ABC. Como será o profissional da indústria 4.0? 2016. Disponível em: https://exame.com/tecnologia/como-sera-o-profissional-da-industria-4-0/. Acesso em 06 ago. 2020.

FERREIRA, Jean Daniel Foro; CONCEIÇÃO, Stéfani Gross; FONSECA, Wellington da Silva. Desenvolvimento de Material Didático Utilizando Realidade Aumentada para Aulas de Eletrônica Analógica. In: COBENGE, XLVII, 2019, Fortaleza-CE. Anais [...]. Brasília: ABENGE, 2019, v. 1, s.p.

FORTUNA, Pablo Rocha et al. Desenvolvimento de Material Potencialmente Significativo Utilizando Impressora 3D e Realidade Aumentada para Apoio ao Ensino da Engenharia Civil: Uma Experiência com Alunos do Ensino Médio e da Graduação. In: COBENGE, XLVII, 2019, Fortaleza-CE. Anais [...]. Brasília: ABENGE, 2019, v. 1, s.p.

FRANÇA, Carlos Roberto; SILVA, Tatiana. A Realidade Virtual e Aumentada e o Ensino de Ciências. Revista de Estudos e Pesquisas sobre Ensino Tecnológico, Manaus, v. 05, n. 10, p.193-215, mar. 2019. Edição especial.

FRANÇA, Carlos Roberto; SILVA, Tatiana. A realidade virtual e aumentada dedicada ao processo ensino-aprendizagem de física: socialização da concepção e validação do aplicativo RVA_360 - Momento Angular. Revista Brasileira de Ensino de Ciência e Tecnologia, Ponta Grossa, v.13, n. 1, p. 142-169, jan./abr. 2020. Disponível em: https://periodicos.utfpr.edu.br/rbect. Acesso em: 27 jul. 2020. 
GOMES JUNIOR, Wedson; CARDOSO, Alexandre; LAMOUNIER JUNIOR, Edgard. Ferramenta de Auxílio ao Aprendizado de Motores com o Uso de Realidade Virtual. Anais do VII Workshop de Realidade Virtual e Aumentada (WRVA), 2010, São Paulo, p. 37 41 .

HAMILTON, Karen E., \& OLENEWA, Jorge. Augmented reality in education. Proc. SXSW Interactive. 2011. M. Kapur. Productive failure in learning math. Cognitive Science, 38(5):1008-1022, 2014.

HERPICH, Fabricio et al. Atividade educacional utilizando Realidade Aumentada para o Ensino de Física no Ensino Superior. Revista Iberoamericana de Tecnología en Educación y Educación en Tecnología, n. 25, p. e7, 27 jul. 2020.

LEITE, Saulo. et al. Espaço Maker: Aprendizagem Ativa com Alunos do ensino Fundamental e Médio do Sistema de Ensino Equipe. In: COBENGE, XLVII, 2019, Fortaleza-CE. Anais [...]. Brasília: ABENGE, 2019, v. 1, s.p.

LIMA, Marcelo Bernardo et al. Realidade aumentada no ensino de ciência: uma revisão de literatura. In: XI Encontro Nacional de Pesquisa em Educação em Ciência (ENPEC). Universidade Federal de Santa Catarina 2017, Florianópolis/SC. Anais [...]. Florianópolis, 2017.

LIMA, Mayanne de Oliveira et al. Uso da Realidade Aumentada (RA) como Ferramenta Facilitadora no Ensino de Disciplinas de Estruturas. In: COBENGE, XLVII, 2019, FortalezaCE. Anais [...]. Brasília: ABENGE, 2019, v. 1, s.p.

LOPES, Luana Monique Delgado et al. INOVAÇÕES EDUCACIONAIS COM O USO DA REALIDADE AUMENTADA: uma revisão sistemática. Educação em Revista, [S.L.], v. 35, p. 1-33, 2019. FapUNIFESP (SciELO). http://dx.doi.org/10.1590/0102-4698197403

LORENZ, Markus et al. Industry 4.0: The Future of Productivity and Growth in Manufacturing Industries. 2015. Disponível em: https://www.bcg.com/ptbr/publications/2015/engineered_products_project_business_industry_4_future_productivity_ growth_manufacturing_industries. Acesso em: 03 ago. 2020.

MARTINS, Valéria Farinazzo; OLIVEIRA, Alisson José Gregório; GUIMARÃES, Marcelo Paiva. Implementação de um laboratório de realidade virtual de baixo custo: estudo de caso de montagem de um laboratório para o ensino de Matemática. Revista Brasileira de Computação Aplicada, v. 5, n. 1, p. 98-112, 17 maio 2013.

MERINO, Cristian et al. O ensino do modelo atômico de Borh em livros texto e uma nova proposta com realidade aumentada para promover a visualização. Revista Brasileira de Ensino de Ciência e Tecnologia, v. 11, n. 2, 2018. Disponível em: https://periodicos.utfpr.edu.br/rbect/article/view/8453. Acesso em: 30 jul. 2020.

MOORE, Michael G. Theoretical principles of distance education. Theoretical principles of distance education, London: Routledge. p. 22-39, 1993. 
OLIVEIRA, Danieli Soares et al. Desenvolvimento de Ambiente de Realidade Virtual para Ensino/Capacitação Técnica na Área de Manutenção Eletromecânica Ferroviária. In: COBENGE, XLVII, 2019, Fortaleza-CE. Anais [...]. Brasília: ABENGE, 2019, v. 1, s.p.

OLIVEIRA, Luciano Denardin; MANZANO, Ramón Cid. Aplicações de realidade aumentada no ensino de Física a partir do software LAYAR. Revista Novas Tecnologias na Educação, [S.L.], v. 14, n. 1, 26 ago. 2016. Universidade Federal do Rio Grande do Sul. http://dx.doi.org/10.22456/1679-1916.67326

PARCIANELLO, Yussef; LEMOS II, Dalton Luiz. Utilização de Realidade Aumentada Buscando Maior Engajamento dos Alunos no Ensino de Desenho Técnico e Desenho Assistido por Computador. In: COBENGE, XLVI, 2018, Salvador-BA. Anais [...]. Brasília: ABENGE, 2018, v. 1, s.p.

PAVANI, Sergio Adalberto et al. Novas tecnologias no ensino técnico/tecnológico. In: Congresso Brasileiro de Educação em Engenharia (COBENGE), XLV, 2017, Joinville-SC. Anais [...]. Brasília: ABENGE, 2017, v. 1, s.p.

PAVANI, Sergio Adalberto; POZZER, Sergio Adalberto; COLUSSO, Sergio Adalberto. Bancada Virtual para Laboratórios de Automação Pneumática, Hidráulica, Acionamento de Motores E Controladores de Processo - Um Caso em Evolução. In: COBENGE, XLVII, 2019, Fortaleza-CE. Anais [...]. Brasília: ABENGE, 2019, v. 1, s.p.

QUEIROZ, Anna Carolina; TORI, Romero; NASCIMENTO, Alexandre. Realidade Virtual na Educação: panorama das pesquisas no brasil. Anais do XXVIII Simpósio Brasileiro de Informática na Educação (Sbie 2017), [S.L.], p. 203-212, 27 out. 2017. Brazilian Computer Society (Sociedade Brasileira de Computação - SBC).

http://dx.doi.org/10.5753/cbie.sbie.2017.203

QUEIROZ, Eduarda; MOURA, Rafaela; SOUZA Ellen. Como a Realidade Aumentada tem Auxiliado no Processo de Ensino e Aprendizagem de Ciências da Natureza? Um Mapeamento Sistemático da Literatura. In: IV Congresso sobre Tecnologias na Educação (Ctrl+E), 2019, Recife-PE, Anais [...]. Recife-PE, 2019.

ROLIM, Ana Caroline Uliana et al. Utilização de Técnicas de Mapeamento de Processos para o Desenvolvimento de Plataforma de Realidade Virtual para Ensino/Treinamento na Área de manutenção Eletromecânica Ferroviária. In: COBENGE, XLVII, 2019, Fortaleza-CE. Anais [...]. Brasília: ABENGE, 2019, v. 1, s.p.

SANTOS, Ricardo Silva et al. Realidade Aumentada no Processo de Ensino-Aprendizagem da Topografia em Projeto de Engenharia Civil. In: III Congresso sobre Tecnologias na Educação (Ctrl+E), 2018, Fortaleza-CE, Anais [...]. Fortaleza-CE, 2018.

SCORTEGAGNA, Luan F.; GONÇALVES, Flávia A. Utilização de Realidade Aumentada na Otimização do Ensino-aprendizagem de Desenho Técnico na Engenharia. In: COBENGE, XLVI, 2018, Salvador-BA. Anais [...]. Brasília: ABENGE, 2018, v. 1, s.p.

SENA, Manoel; ROCHA, Paulo Santana; NASCIMENTO, Raul Batista. Simulador Virtual 3D para Atividades Práticas de Aprendizado em Análise de Vibrações. In: COBENGE, XLVI, 2018, Salvador-BA. Anais [...]. Brasília: ABENGE, 2018, v. 1, s.p. 
SILVA, George Geraldo de Oliveira et al. Indústria 4.0: Um Estudo sobre sua Aplicação no Ensino Técnico Profissionalizante. Revista Brasileira de Mecatrônica, São Caetano do Sul, v. 2, n.1, p.14-27, jul./set. 2019.

SILVA, João et al. Adoção de Realidade Aumentada no Ensino de Resistência dos Materiais. Anais dos Workshops do IV Congresso Brasileiro de Informática na Educação (Cbie 2015), [S.L.], p. 1198-1207, 26 out. 2015. Sociedade Brasileira de Computação - SBC. http://dx.doi.org/10.5753/cbie.wcbie.2015.1198

SILVA, Kássio Vinícius.; SÁ JÚNIOR, José Carlos; AZEVÊDO, Wilker Victor da Silva. Sobre Concepções no Estudo de Circuitos Elétricos e utilização de Experimentação Real e Virtual. In: COBENGE, XLVI, 2018, Salvador-BA. Anais [...]. Brasília: ABENGE, 2018, v. 1, s.p.

VIEGAS, Márcio A. C.; VIEIRA, Marcelo B.; SILVA, Rodrigo Luis de S. Ferramenta de Apoio ao ensino de Física utilizando Realidade Aumentada. Revista Brasileira de

Informática na Educação, [S.L.], v. 20, n. 03, p. 60-73, 1 dez. 2012. Sociedade Brasileira de Computação - SB. http://dx.doi.org/10.5753/rbie.2012.20.03.60

SILVA, Sérgio Ricardo Xavier. Utilização de um Laboratório Virtual de Física Através de Physlets para Cursos de Engenharia. In: COBENGE, XLVII, 2019, Fortaleza-CE. Anais [...]. Brasília: ABENGE, 2019, v. 1, s.p.

SOUSA, Stephanie Kamarry Alves et al. Realidade Virtual e Aumentada: Apoio para a Prática Contextualizada e Interdisciplinas na Educação Básica, Técnica e Tecnológica.

Revista Expressão Científica - Edição Especial SNCT [e-book], 2019. Instituto Federal de Sergipe - ano. 4, v.4, n³ Aracaju: IFS, 2019.

SOUZA, Adysson André Fortuna; MORAIS, Maic Araújo da Conceição; CARVALHO, Márcio Aurélio Matos. Utilização da Realidade Aumentada para Facilitar na Visualização de Exercícios Tridimensionais de Mecânica Vetorial. In: COBENGE, XLVI, 2018, SalvadorBA. Anais [...]. Brasília: ABENGE, 2018, v. 1, s.p.

STANGE, Folker; SCHEER, Sérgio. Utilização de Técnicas de Realidade Virtual e Visualização Científica para Desenvolvimento de um Ambiente de Treinamento Técnico na Construção Civil. Proceedings Of 10Th World Congress On Computational Mechanics, [S.L.], maio 2014. Editora Edgard Blücher. http://dx.doi.org/10.5151/meceng-wccm2012$\underline{20118}$

TORI, Romero; HOUNSELL, Marcelo da Silva. Introdução a realidade virtual e aumentada. Porto Alegre: Editora SBC, 2018.

TORI, Romero; KIRNER, Claudio; SISCOUTTO, Robson. Fundamentos e tecnologia de realidade virtual e aumentada. Editora SBC, 2018.

VILAR, Sammara Raquel; ARAÚJO, Lincoln Machado; COSTA JUNIOR, Ademar Gonçalves. Desenvolvimento de Ambiente Virtual de Simulação para Auxiliar o Processo de Ensino-aprendizagem de Robótica na Engenharia. In: COBENGE, XLV, 2017, Joinville-SC. Anais [...]. Brasília: ABENGE, 2017, v. 1, s.p. 


\section{Agradecimentos}

Agradecemos ao professor Me. Cláudio Alves Pereira pela orientação e convívio durante a realização deste trabalho. Agradecemos também o apoio dos campi Sabará e Arcos do Instituto Federal de Educação, Ciência e Tecnologia de Minas Gerais (IFMG) ao trabalho desenvolvido. 